\section{Case Reports in Acute Medicine}

Case Rep Acute Med 2020;3:46-52

DOI: 10.1159/000509569

Published online: August 25, 2020 (c) 2020 The Author(s)

Published by S. Karger AG, Basel www.karger.com/cra

This article is licensed under the Creative Commons Attribution-NonCommercial 4.0 International License (CC BY-NC) (http://www.karger.com/Services/OpenAccessLicense). Usage and distribution for commercial purposes requires written permission.

\title{
Artery of Percheron Infarct: A Rare Presentation of Acute Ischemic Stroke in a High-Risk Antiphospholipid Syndrome Patient
}

\author{
Sreethish Sasi ${ }^{a} \quad$ Ashraf Ahmed $^{a} \quad$ Wajiha Yousuf $^{b} \quad$ Surjith Vattoth \\ aDepartment of Internal Medicine, Hamad General Hospital, Hamad Medical Corporation, \\ Doha, Qatar; 'Weil Cornell Medical College, Doha, Qatar; 'Department of Radiology, \\ Hamad Medical Corporation, Doha, Qatar
}

\section{Keywords}

Thalamus · Artery of Percheron · Amnesia $\cdot$ Antiphospholipid syndrome

\begin{abstract}
Artery of Percheron (AOP) is an abnormal variant of the arterial supply of the thalamus. AOP occlusion can lead to bilateral thalamic and rostral midbrain infarct presenting as memory loss, fluctuating levels of consciousness, and altered mental status. A 43-year-old woman with a history of antiphospholipid syndrome (APS), managed on dabigatran, presented with acute confusion and drowsiness. She had slurred and slowed speech, disorientation in time and place, left-sided facial droop, decreased power of the left side (4/5), and was unable to walk due to generalized weakness. Labs showed a prolonged prothrombin time and activated partial thromboplastin time, positive lupus anticoagulant, anti-cardiolipin, beta-2 glycoprotein, anti-nuclear and anti-dsDNA antibodies. Contrast-enhanced CT perfusion showed ischemic changes in the bilateral thalami, suggesting infarct along the AOP territory. AOP infarcts are scarce and the presenting complaints are unusual of cerebrovascular accidents. It requires a high index of suspicion to detect. There are no other reports in the literature of patients with
\end{abstract}




\section{Case Reports in Acute Medicine}

Case Rep Acute Med 2020;3:46-52

DOI: $10.1159 / 000509569$

(c) 2020 The Author(s). Published by S. Karger AG, Basel www.karger.com/cra

Sasi et al.: Artery of Percheron Infarct in Antiphospholipid Syndrome

APS presenting with an AOP infarct. Considering the lack of evidence, we recommend against the use of newer oral anticoagulants (NOACs) for secondary prevention of vascular events in patients with triple-positive APS.

(C) 2020 The Author(s)

Published by S. Karger AG, Basel

\section{Introduction}

The arterial supply of the thalamus may show an abnormal variant in 33\% of humans, known as AOP. It is a rare occurrence that occlusion of a single artery can cause infarction in bilateral structures [1]. The clinical presentation of such infarcts is unique and different from ischemic infarcts involving other arteries such as the middle cerebral artery (MCA). The symptoms involve alterations in normal functions of the thalamus [2]. AOP is characterized by a single perforating artery that arises from the P1 segment of the posterior cerebral artery between the basilar and posterior communicating arteries. The significance of this artery is that it supplies paramedian thalamus and rostral midbrain on both sides. The main functions of the thalamus include emotions, memory, alertness, sleep-wake cycle, and sensorimotor control [3]. Ischemic strokes usually present with clinically recognizable focal neurological deficits or syndromes. In contrast, an infarct in the AOP territory presents with non-specific symptoms such as memory loss, fluctuating level of consciousness, and altered mental status [4]. The early recognition of these symptoms needs a high index of suspicion, especially in highrisk patients. Here, we present the clinical case of a young lady with a known history of highrisk antiphospholipid syndrome (APS) who presented with confusion and drowsiness, due to bilateral thalamic stroke caused by infarction along the AOP.

\section{Case Presentation}

A 43-year-old woman with a history of APS, managed on dabigatran, presented to the emergency department after her husband found her confused and drowsy on waking from sleep. She had slurred speech and was unable to walk due to generalized weakness. Her symptoms were not associated with dizziness, headache, vomiting, or nausea. She denied abnormal movements or seizures. Review of systems was unremarkable. Her past medical history was remarkable for a right MCA stroke 2 years back, resulting in a mild residual left-sided facial droop and APS diagnosed during evaluation of the stroke. She was treated with apixaban for 1 year, followed by dabigatran $150 \mathrm{mg}$ twice a day as per recommendation from her neurologist in the UK. She had no history of obstetric complications or rheumatological conditions. Her family history was also unremarkable. On physical exam, she had fluctuating consciousness and was disoriented to time and place. She also had slowed speech, left-sided facial droop, and mildly decreased left upper and lower limb power (4/5). She had no evidence of other focal neurological deficits or nystagmus. CBC and CMP were unremarkable. Blood cultures, urine cultures and toxicology screens were negative. Coagulation studies showed prolonged PT and aPTT. Labs to confirm her diagnosis were positive for lupus anticoagulant, anticardiolipin antibodies, beta-2 glycoprotein, anti-nuclear antibodies, and anti-dsDNA antibodies. Nonenhanced CT head done at the time of admission demonstrated no acute ischemic or 


\section{Case Reports in Acute Medicine}

Case Rep Acute Med 2020;3:46-52

DOI: $10.1159 / 000509569$

(c) 2020 The Author(s). Published by S. Karger AG, Basel www.karger.com/cra

Sasi et al.: Artery of Percheron Infarct in Antiphospholipid Syndrome

hemorrhagic changes, but there was a chronic infarct with encephalomalacia in the right frontoparietal region MCA territory. Contrast-enhanced CT perfusion scan done at the same setting showed ischemic changes in the bilateral thalami, with a small focal infarct within the left thalamic ischemia, suggesting occlusion of the AOP (Fig. 1). A diffusion-weighted MRI done after $4 \mathrm{~h}$ showed diffusion restriction consistent with acute infarct in the left thalamic focal area of infarct with low CBV seen in CT perfusion, and a subtle tiny focus of evolving hyperacute infarct in the right thalamus (Fig. 2). There were also tiny acute infarcts in the right side of the midbrain and a right MCA territory chronic infarct with encephalomalacia. Follow-up MRI done after 2 days demonstrated the expected evolution of the acute thalamic and right midbrain infarcts. MR arteriogram showed attenuated right MCA branches in the region of the chronic infarct. The patient remained in the hospital for 6 days, and on discharge, she was oriented to time, person, and place. Her speech was no longer slowed. She regained full motor power but had minor lapses in memory that was improving. She was discharged on warfarin for secondary prevention and did not report any vascular or embolic events at the 3-month follow-up.

\section{Discussion}

The thalamus is a bilateral, oval-shaped structure which is located deep in the grey matter. The main functions of the thalamus include emotions, memory, alertness, sleep-wake cycle, and sensorimotor control. Intralaminar nuclei of the thalamus may project into the brainstem ascending reticular activating system (ARAS) or cerebellum, and an infarct here presents with coma or cerebellar signs, respectively. The involvement of mediodorsal and anterior nuclei results in memory impairment, aphasia, dysarthria, and psychosis, whereas the involvement of rostral midbrain results in frontal eye field defects $[2,4]$. Anatomically, there is a rare variation, in which the rostral midbrain and paramedian thalami are bilaterally supplied by a solitary arterial trunk in the posterior cerebral circulation. This is known as AOP. There could be three types of such variation namely, type 1 in which one perforating artery arises from each PCA and each one supplies bilateral thalami, type 2 in which a single perforating artery arises from the PCA and supplies bilateral thalami, and type 3 in which one perforating artery arises from each PCA, and these are connected by a communicating artery to supply bilateral thalami [5]. A close differential of thalamic infarction is Korsakoff syndrome with similar clinical and imaging findings. As their treatments are different, it becomes essential to differentiate them neuropsychologically, which can be done based on features mentioned in Table 1 [6]. AOP infarctions usually cannot be picked up in CT imaging. CT angiography is not useful as the vessels involved are of small caliber [2]. MRI is diagnostic in $100 \%$ of cases [5]. There might be a delay in the diagnosis of patients with this type of stroke for several hours considering the vague neurological features with which they present.

Low-dose aspirin (LDA) is recommended for primary prevention in patients with a highrisk antiphospholipid antibody (aPL) profile, even without clinically diagnosed APS. High-risk aPL profiles refer to the presence of lupus anticoagulant at least two times 12 weeks apart and persistently positive aPL with double or triple titer [7]. Secondary prevention with vitamin K antagonist (VKA) targeting INR of 2-3 is recommended for patients with APS who had previous venous thromboembolism (VTE) or arterial thrombosis. VKA is recommended over LDA. 


\section{Case Reports in Acute Medicine}

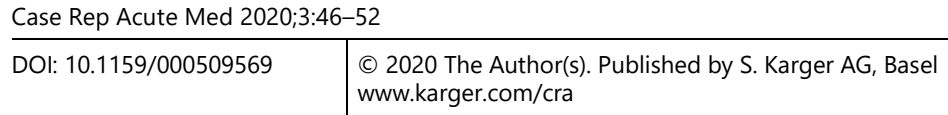
www.karger.com/cra

Sasi et al.: Artery of Percheron Infarct in Antiphospholipid Syndrome

However, in patients with recurrent VTE or arterial thrombosis despite VKA with target INR of 2-3, adding LDA or increasing the INR target to 3-4 or switching to LMWH may be considered [8]. The role of newer oral anticoagulants (NOACs) in APS is not well studied. A randomized control trial (RCT) to compare rivaroxaban with warfarin had more recurrent thromboses in the rivaroxaban group. The events were predominantly arterial, with a recurrent stroke occurring in $10 \%$ of patients receiving rivaroxaban versus none of the patients receiving warfarin [9]. Another RCT comparing rivaroxaban with warfarin in triple antibody positive APS was aborted because of the increased rate of arterial events in the rivaroxaban arm [10]. A systematic review of patients with APS treated with NOACs identified that $15.5 \%$ of patients experienced a recurrent thrombosis, with triple antibody positivity being associated with a 3.5-fold increased risk [11]. Physicians who prescribe NOACs for APS patients depend on case reports, post-hoc analysis, and personal experience as there is no clear evidence recommending NOACs over VKA. NOACs seem to be an option for those who are not compliant with VKA or not tolerating it. Still, the patient should be informed regarding the higher risk of recurrence of venous and arterial thrombotic events. NOACs should never be used for secondary prevention in patients with triple-positive APS. Our patient had triple-positive APS, and the use of dabigatran did not protect her against the recurrence of an arterial thrombotic event.

\section{Conclusion}

AOP infarcts are rare and often misdiagnosed as the initial radiological evaluations are usually normal. Symptoms of AOP are different from other ischemic strokes and vary depending on the size and distribution of the infarct. A sudden onset alteration in sensorium with behavioral manifestation and abnormal eye movements are clues suggestive of an AOP infarction. A diffusion-weighted MRI of the brain in the early hours is the investigation of choice. A detailed evaluation of cardiac and arterial sources of embolism is recommended in all cases of AOP infarction.

\section{Acknowledgments}

The authors would like to acknowledge the Internal Medicine Residency Program at Hamad Medical Corporation for scientific support.

\section{Statement of Ethics}

The case was approved by the Medical Research Center at Hamad Medical Corporation.

Written informed consent was obtained from the patient to publish her case information and details. 
Case Reports in
Acute Medicine

Case Rep Acute Med 2020;3:46-52

DOI: $10.1159 / 000509569$

(C) 2020 The Author(s). Published by S. Karger AG, Basel www.karger.com/cra

Sasi et al.: Artery of Percheron Infarct in Antiphospholipid Syndrome

\section{Conflict of Interest Statement}

The authors have no conflicts of interest to declare.

\section{Funding Sources}

The publication of this article was funded by the Qatar National Library.

\section{Author Contributions}

S.S.: Concept, manuscript preparation, manuscript editing, literature search, manuscript review, and patient management. He will act as a study guarantor. A.A.: Literature search, manuscript preparation, and patient management. W.Y.: Manuscript preparation and editing. S.V.: Manuscript review and editing, Critical inputs in radiology and images. All authors have reviewed and agreed on the manuscript.

\section{References}

1 Caruso P, Manganotti P, Moretti R. Complex neurological symptoms in bilateral thalamic stroke due to Percheron artery occlusion. Vasc Health Risk Manag. 2016 Dec;13:11-4.

2 Khanni JL, Casale JA, Koek AY, Espinosa Del Pozo PH, Espinosa PS. Artery of Percheron Infarct: An Acute Diagnostic Challenge with a Spectrum of Clinical Presentations. Cureus. 2018 Sep;10(9):e3276.

3 Castaigne P, Lhermitte F, Buge A, Escourolle R, Hauw JJ, Lyon-Caen O. Paramedian thalamic and midbrain infarct: clinical and neuropathological study. Ann Neurol. 1981 Aug;10(2):127-48.

4 Chen XY, Wang Q Wang X, Wong KS. Clinical Features of Thalamic Stroke. Curr Treat Options Neurol. 2017 Feb;19(2):5.

$5 \mathrm{Xu}$ Z, Sun L, Duan Y, Zhang J, Zhang M, Cai X. Assessment of Percheron infarction in images and clinical findings. J Neurol Sci. 2017 Dec;383:87-92.

6 Kopelman MD. What does a comparison of the alcoholic Korsakoff syndrome and thalamic infarction tell us about thalamic amnesia? Neurosci Biobehav Rev. 2015 Jul;54:46-56.

7 Tektonidou MG, Andreoli L, Limper M, Amoura Z, Cervera R, Costedoat-Chalumeau N, et al. EULAR recommendations for the management of antiphospholipid syndrome in adults. Ann Rheum Dis. 2019 Oct;78(10):1296-304.

8 Finazzi G, Marchioli R, Brancaccio V, Schinco P, Wisloff F, Musial J, et al. A randomized clinical trial of highintensity warfarin vs. conventional antithrombotic therapy for the prevention of recurrent thrombosis in patients with the antiphospholipid syndrome (WAPS). J Thromb Haemost. 2005 May;3(5):848-53.

9 Ordi-Ros J, Sáez-Comet L, Pérez-Conesa M, Vidal X, Riera-Mestre A, Castro-Salomó A, et al. Rivaroxaban Versus Vitamin K Antagonist in Antiphospholipid Syndrome: A Randomized Noninferiority Trial. Ann Intern Med. 2019 Nov;171(10):685-94.

10 Pengo V, Denas G, Zoppellaro G, Jose SP, Hoxha A, Ruffatti A, et al. Rivaroxaban vs warfarin in high-risk patients with antiphospholipid syndrome. Blood. 2018 Sep;132(13):1365-71.

11 Dufrost V, Risse J, Zuily S, Wahl D. Direct Oral Anticoagulants Use in Antiphospholipid Syndrome: Are These Drugs an Effective and Safe Alternative to Warfarin? A Systematic Review of the Literature. Curr Rheumatol Rep. 2016 Dec;18(12):74. 


\section{Case Reports in Acute Medicine}

Case Rep Acute Med 2020;3:46-52

DOI: $10.1159 / 000509569$

(C) 2020 The Author(s). Published by S. Karger AG, Basel www.karger.com/cra

Sasi et al.: Artery of Percheron Infarct in Antiphospholipid Syndrome

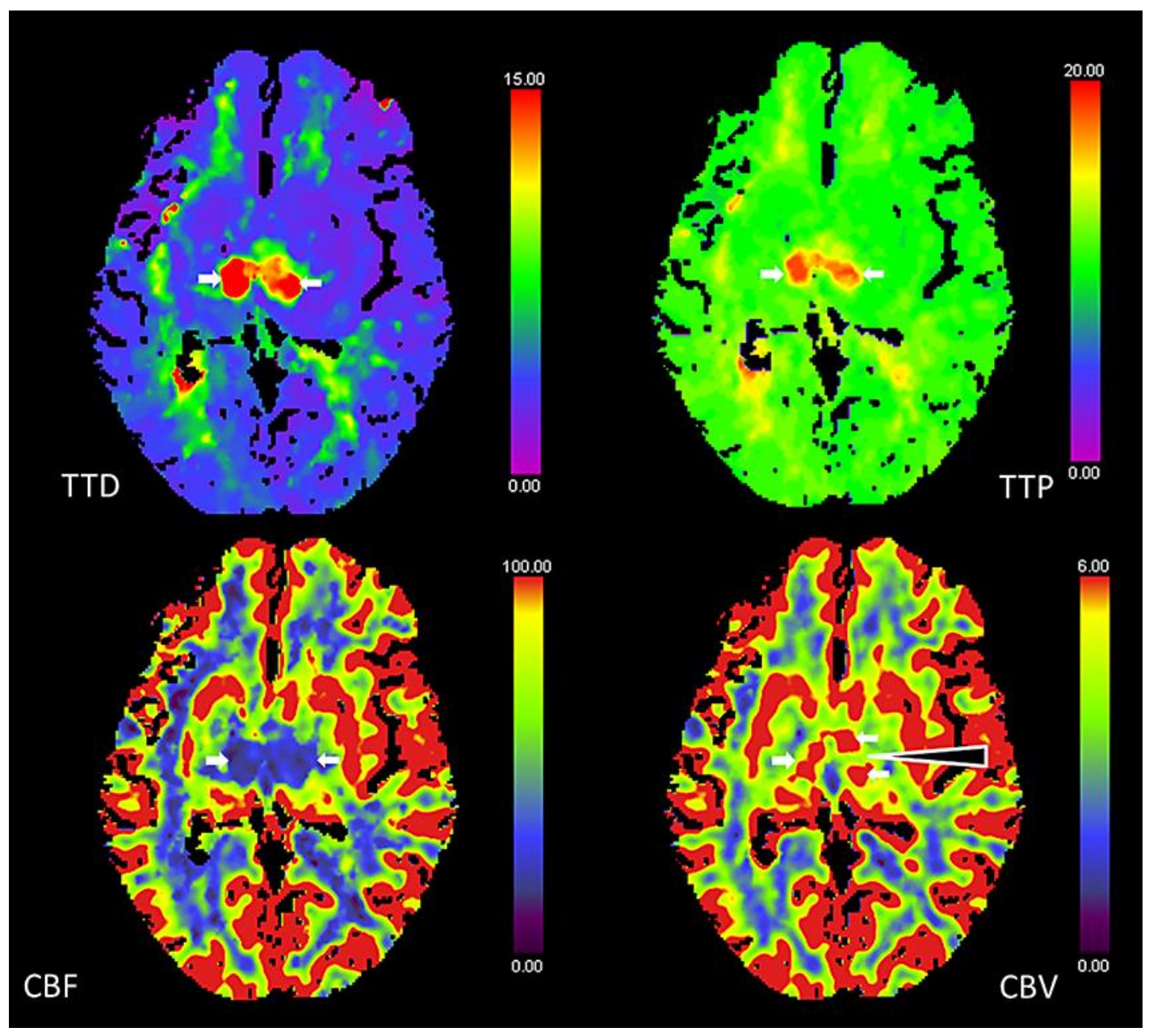

Fig. 1. CT perfusion study on admission showing bilateral thalamic increased time to drain (TTD) and time to peak (TTP) in the color parametric maps seen as red color (arrows). Reduced cerebral blood flow (CBF) seen as blue color (arrows) consistent with ischemia. The cerebral blood volume (CBV) is reduced focally within the center of the left thalamic perfusion abnormality, seen as green color (long arrowhead), suggesting infarction within this focal area of the ischemic thalami. Reminder of the thalami shows maintained CBV seen as red color (arrows), which suggests ischemic penumbra. 


\section{Case Reports in Acute Medicine}
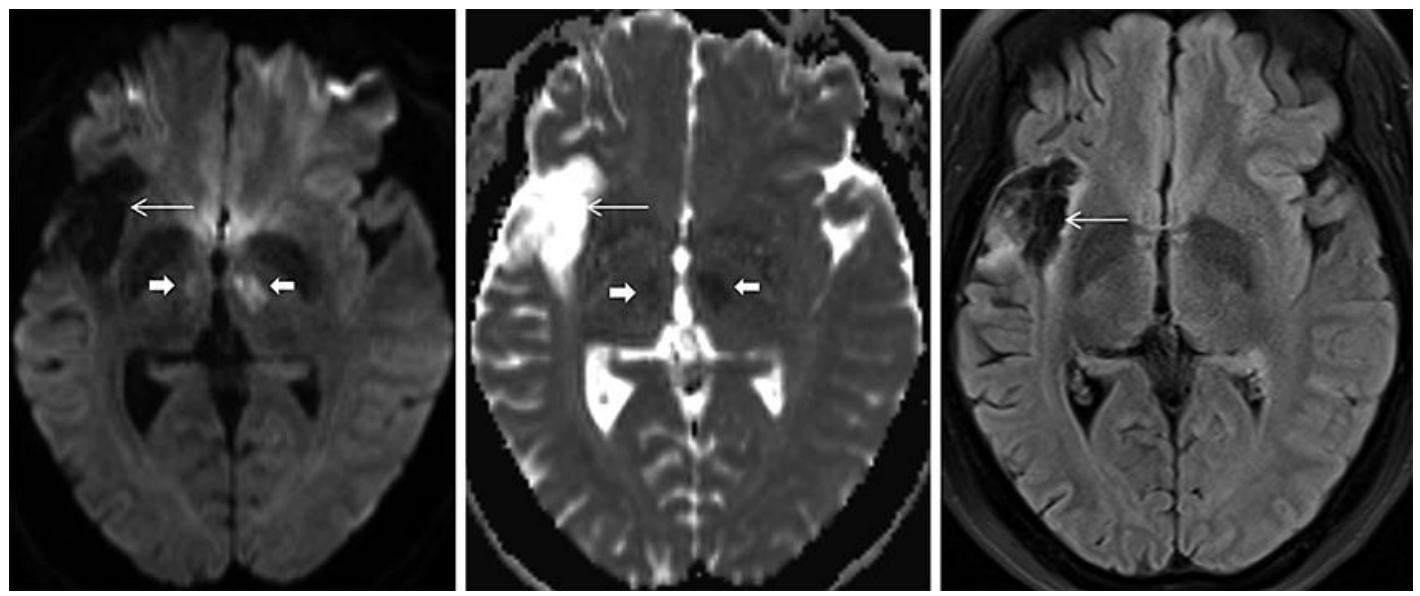

Fig. 2. Diffusion-weighted MRI done after $4 \mathrm{~h}$ of the CT perfusion scan showing diffusion restriction (arrow) consistent with acute infarct in the left thalamic focal area of infarct showing low CBV seen in the CT perfusion scan. There is also a subtle tiny focus of evolving hyperacute infarct in the right thalamus (arrow). Apparent diffusion coefficient (middle image) confirms diffusion restriction with corresponding hypointensity in the infarcts (arrow). FLAIR (right image) does not show any signal abnormality because of the hyperacute stage of the infarct. Note the non-diffusion restricting right MCA territory chronic infarct with encephalomalacia in all images (thin arrow).

Table 1. Clinical features to distinguish between Korsakoff syndrome and thalamic infarction

\begin{tabular}{lll}
\hline & Korsakoff syndrome & Thalamic infarction \\
\hline Recall/recollection & $\square \square \square$ & $\square \square \square$ \\
Recognition/familiarity & $\square \square$ & $\square /$ spared \\
Context memory & $\square \square$ & Not well studied \\
Anterograde amnesia & $\square \square$ & $\square \square \square$ \\
Retrograde amnesia & $\square \square$ & $\square /$ spared \\
Spontaneous confabulation & Extensive (up to 25 years) & Less frequent, less extensive (0-10 years) \\
\hline
\end{tabular}

